\title{
A PET imaging agent for evaluating PARP-1 expression in ovarian cancer
}

\author{
Mehran Makvandi, ${ }^{1}$ Austin Pantel, ${ }^{1}$ Lauren Schwartz, ${ }^{2}$ Erin Schubert, ${ }^{1}$ Kuiying Xu, ${ }^{1}$ Chia-Ju Hsieh, ${ }^{1}$ Catherine Hou, ${ }^{1}$ \\ Hyoung Kim, ${ }^{3}$ Chi-Chang Weng, ${ }^{1}$ Harrison Winters, ${ }^{4}$ Robert Doot, ${ }^{1}$ Michael D. Farwell, ${ }^{1}$ Daniel A. Pryma, ${ }^{1}$ Roger A. Greenberg, ${ }^{4}$ \\ David A. Mankoff, ${ }^{1}$ Fiona Simpkins, ${ }^{3}$ Robert H. Mach, ${ }^{1}$ and Lilie L. Lin ${ }^{5}$

\begin{abstract}
'Department of Radiology, Division of Nuclear Medicine and Molecular Imaging, University of Pennsylvania, Perelman School of Medicine, Philadelphia, Pennsylvania, USA. ²Department of Pathology, Hospital of the University of Pennsylvania, Philadelphia, Pennsylvania, USA. ${ }^{3}$ Department of OBGYN, Division of Gynecology and Oncology, ${ }^{4}$ Department of Cancer Biology, and ${ }^{5}$ Department of Radiation Oncology, University of Pennsylvania, Perelman School of Medicine, Philadelphia, Pennsylvania, USA.
\end{abstract}

\begin{abstract}
BACKGROUND. Poly(ADP-ribose) polymerase (PARP) inhibitors are effective in a broad population of patients with ovarian cancer; however, resistance caused by low enzyme expression of the drug target PARP-1 remains to be clinically evaluated in this context. We hypothesize that PARP-1 expression is variable in ovarian cancer and can be quantified in primary and metastatic disease using a novel PET imaging agent.
\end{abstract}

\begin{abstract}
METHODS. We used a translational approach to describe the significance of PET imaging of PARP-1 in ovarian cancer. First, we produced PARP1-KO ovarian cancer cell lines using CRISPR/Cas9 gene editing to test the loss of PARP-1 as a resistance mechanism to all clinically used PARP inhibitors. Next, we performed preclinical microPET imaging studies using ovarian cancer patient-derived xenografts in mouse models. Finally, in a phase I PET imaging clinical trial we explored PET imaging as a regional marker of PARP-1 expression in primary and metastatic disease through correlative tissue histology.
\end{abstract}

RESULTS. We found that deletion of PARP1 causes resistance to all PARP inhibitors in vitro, and microPET imaging provides proof of concept as an approach to quantify PARP-1 in vivo. Clinically, we observed a spectrum of standard uptake values (SUVs) ranging from 2-12 for PARP-1 in tumors. In addition, we found a positive correlation between PET SUVs and fluorescent immunohistochemistry for PARP-1 $\left(r^{2}=0.60\right)$.

CONCLUSION. This work confirms the translational potential of a PARP-1 PET imaging agent and supports future clinical trials to test PARP-1 expression as a method to stratify patients for PARP inhibitor therapy.

TRIAL REGISTRATION. Clinicaltrials.gov NCT02637934.

FUNDING. Research reported in this publication was supported by the Department of Defense OC160269, a Basser Center team science grant, NIH National Cancer Institute R01CA174904, a Department of Energy training grant DE-SC0012476, Abramson Cancer Center Radiation Oncology pilot grants, the Marsha Rivkin Foundation, Kaleidoscope of Hope Foundation, and Paul Calabresi K12 Career Development Award 5K12CA076931.

\section{Introduction}

Epithelial ovarian cancer (EOC) is the fifth deadliest malignancy in women, with more than $70 \%$ of patients presenting with advanced disease (1). Until the recent emergence of targeted therapeutics, treatment has relied heavily on cytoreductive surgery and platinum/taxane-based chemotherapy (2). Poly(ADP-ribose) polymerase inhibitors (PARPis) have emerged as promising drug candidates for the treatment of EOC. In current clinical practice,

\section{Related Commentary: p. 1727}

Conflict of interest: The authors have declared that no conflict of interest exists. Submitted: October 11, 2017; Accepted: February 28, 2018. Reference information: / Clin Invest. 2018;128(5):2116-2126. https://doi.org/10.1172/JCI97992. patients are commonly selected for PARPi therapy by testing positive for genetic mutations in genes encoding DNA repair proteins that result in homologous recombination deficiency (HRD), most notably mutations in breast and ovarian cancer susceptibility genes 1 and 2 (BRCA1 and BRCA2) (3, 4). However, only approximately $50 \%$ of patients with HRD respond to PARPi therapy (3). Moreover, patients without known HRD have also shown a clinical benefit from PARPis, as seen in recent trials assessing niraparib, olaparib, or rucaparib, as maintenance therapy in platinum-sensitive recurrent ovarian cancer (5-8). Given that not all patients will respond to PARPi therapy, improved clinical tools for predicting which patients will respond are urgently needed.

Numerous clinical trials have led to FDA approval of 3 PARPis since 2014 and there is continued development of 2 additional drugs within this class (9-13). Despite growth in the 
A

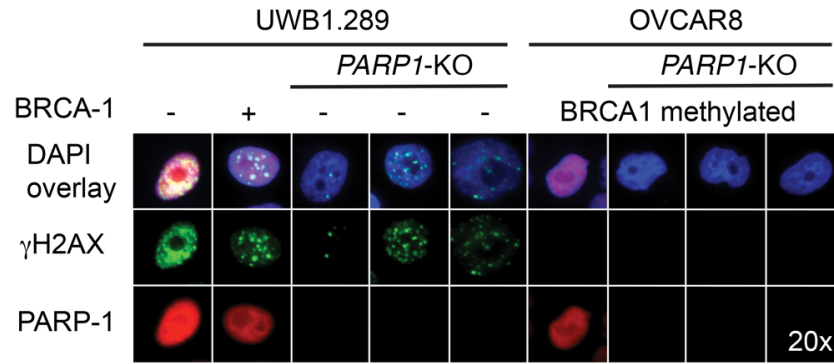

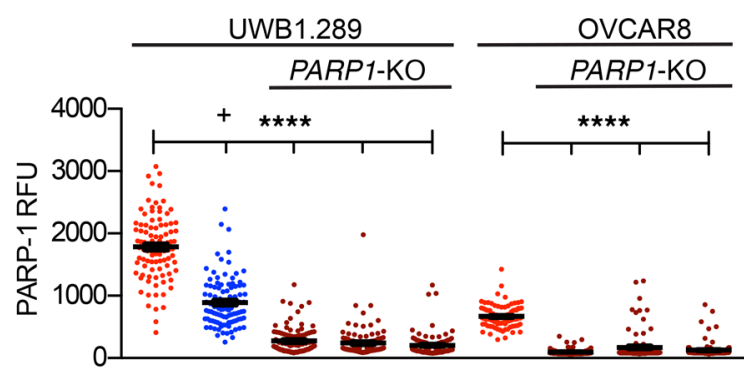

C
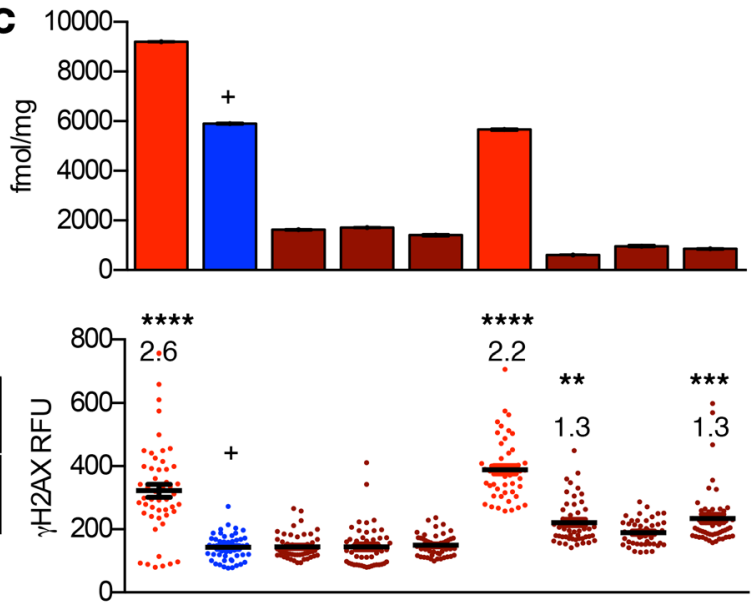

B

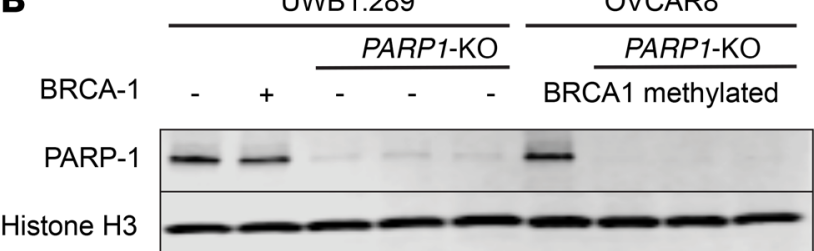

D

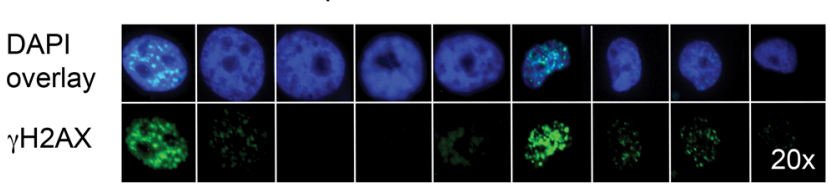

Olaparib treatment for $24 \mathrm{hrs}$
E

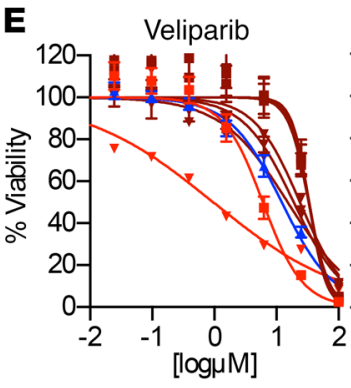

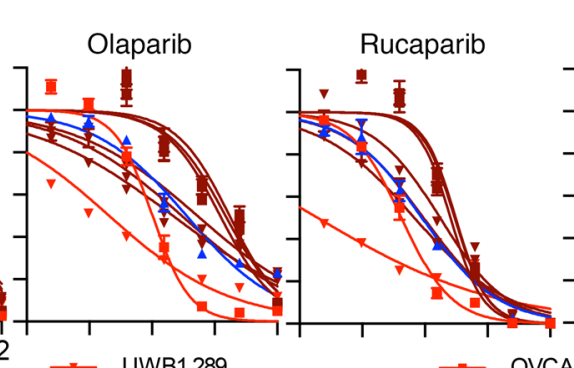
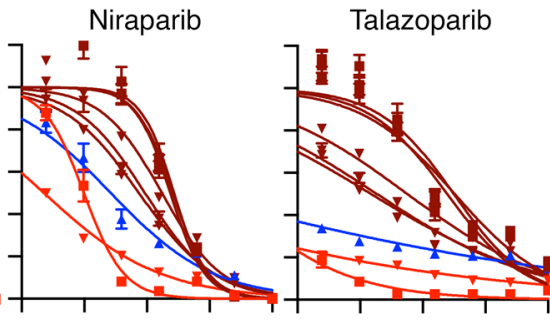

Cisplatin

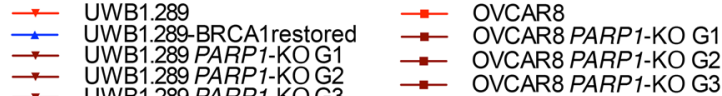
־ UWB1.289 PARP1-KO G2

$\rightarrow-$ OVCAR8 PARP1-KO G2

Figure 1. The characterization of PARP1-KO ovarian cancer cell lines and in vitro evaluation of PARP inhibitor efficacy. (A) Immunofluorescence showed PARP-1 was absent in more than $90 \%$ of single cells in PARP1-KO polyclonal populations (ANOVA, ${ }^{* * *} P<0.0001$ ) and was reduced in BRCA1-restored cells compared with parent control (ANOVA, ${ }^{* * *} P<0.0001$ ). (B) Polyclonal populations of PARP1-KO cell lines had reduced PARP-1 by Western blot compared with parent control. (C) [125]]KX1 radioligand binding assays showed a significant reduction in radiotracer binding in PARP1-KO and UWB1.289 BRCA1restored cell lines compared with parent control (ANOVA, $P<0.0001$ ). (D) Immunofluorescence of olaparib-treated UWB1.289 PARP1-KO and UWB1.289 $B R C A 1$-restored cells showed no increase in $\gamma \mathrm{H} 2 \mathrm{AX}$ compared with DMSO controls. Olaparib-treated OVCAR8 PARP1-KO G1 and G3 cells showed a 1.3 times increase (ANOVA, ${ }^{*} P<0.01$ and ${ }^{* *} P<0.001$, respectively) in $\gamma \mathrm{H} 2 \mathrm{AX}$ from DMSO controls. This was in contrast to olaparib-treated UWB1.289 and OVCAR8 cells that showed a 2.6 times (ANOVA, ${ }^{* * *} P<0.0001$ ) and 2.2 times (ANOVA, ${ }^{* * *} P<0.0001$ ) increase in $\gamma \mathrm{H} 2 \mathrm{AX}$ from DMSO controls. (E) Cell viability assays showed that $P A R P 1-K O$ cells were equally resistant to olaparib compared with BRCA1-restored cells and all clinical PARP inhibitors required PARP-1 for maximum efficacy. Loss of PARP1 caused the greatest change in efficacy for niraparib and talazoparib. Cisplatin sensitivity was used as a positive control and remained unchanged after loss of $P A R P 1$. All in vitro experiments were completed 3 independent times. Cell lines shown in $\mathbf{A}-\mathbf{D}$, from left to right, are: UWB1.289, UWB1.289 BRCA1 restored, UWB1.289 PARP1-KO G1, UWB1.289 PARP1-KO G2, UWB1.289 PARP1-KO G3, OVCAR8, OVCAR8 PARP1KO G1, OVCAR8 PARP1-KO G2, and OVCAR8 PARP1-KO G3. -, BRCA1 mutant. +, BRCA1 restored.

development and application of PARPis, the primary drug target poly(ADP-ribose) polymerase 1 (PARP-1) has never been evaluated in vivo, even though loss of expression in vitro is a wellcharacterized resistance mechanism (3, 14-19). It was first hypothesized that PARPis work primarily through a synthetic lethality pathway where loss of $B R C A 1$ or $B R C A 2$ combined with chemical inhibition of PARP-1 results in cell death $(20,21)$. However, it was later shown that deletion of PARP1 did not result in enhanced sensitivity to a DNA alkylating agent in WT cells, suggesting that loss of catalytic function could not be the only reason for PARPi efficacy (17-19). Next, Murai et al. demonstrated that all clinically used PARPis have differential abilities to trap PARP-1 on DNA, but equally lack cytotoxicity in PARP1-KO-/- cells (22, 23). While some PARPis are potent PARP-1 trappers, all PARPis target the PARP-1 enzyme, making it a potential prerequisite biomarker for efficacy (22-24). Furthermore, PARP-1 has been 
A

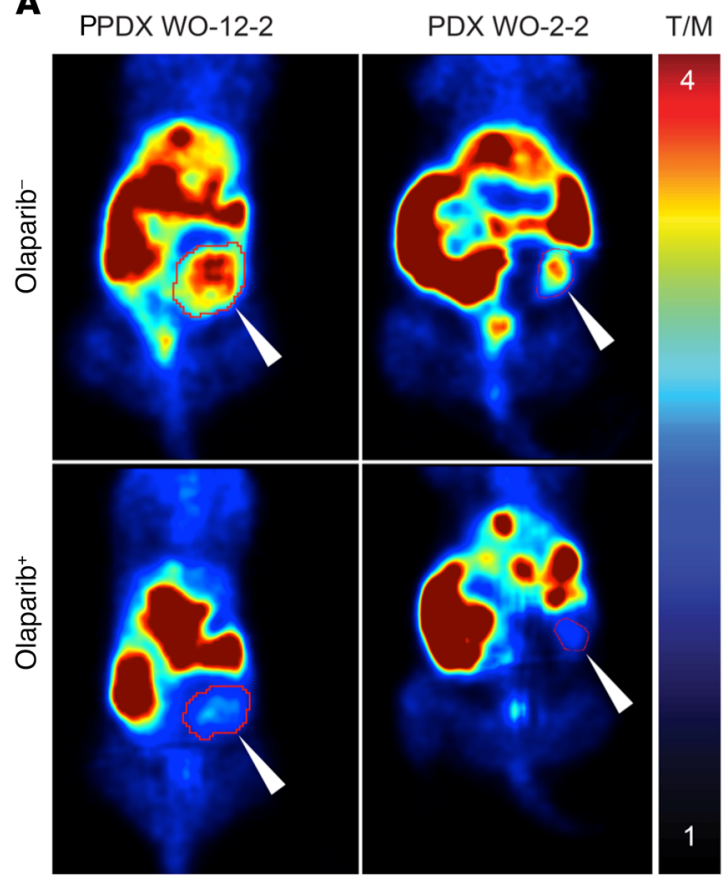

B

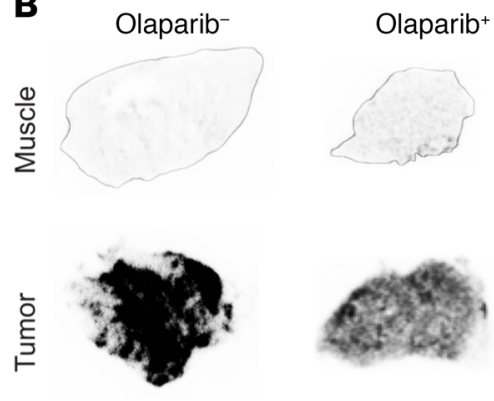

C
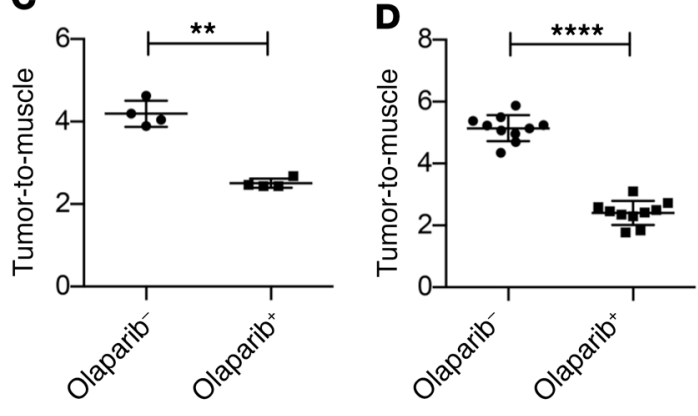

Figure 2. In vivo [ ${ }^{18}$ F]FTT microPET imaging and ex vivo autoradiography of $\mathbf{2}$ xenografts derived from patients with ovarian cancer. (A) Tumor-bearing mice underwent microPET imaging with [ $\left.{ }^{18} \mathrm{~F}\right] \mathrm{FTT}$ before (top images) and after (bottom images) olaparib treatment. White arrows point to patient-derived xenograft tumors. (B) Ex vivo autoradiographs of tumor and muscle from untreated (-) versus olaparib-treated (+) mice. (C) Significant differences were observed in the tumor-to-muscle ratios calculated before and after olaparib treatment from microPET images ( $4.2 \pm 0.32$ vs. $2.5 \pm 0.11$, parametric paired $t$ test, $\left.{ }^{*} P<0.0025, n=4\right)$. (D) Ex vivo autoradiographs of untreated (-) versus olaparib-treated (+) mice shown in $\mathbf{B}$ also showed a statistically significant difference between groups (5.14 \pm 0.13 vs. $2.41 \pm 0.18, n=2,10$ sections/tumor, parametric unpaired $t$ test, ${ }^{* * * *} P<0.0001$ ).

shown by immunohistochemistry (IHC) to be highly variable in patients with ovarian (25-28), breast (29), and prostate cancer (30), irrespective of BRCA status. Together these studies demonstrate the need for a biomarker technology capable of quantitatively assessing PARP-1 in vivo that could enable patient selection for PARPi therapy.

Current methods to determine PARP-1 expression in clinical tumor specimens are limited and based on immunohistological methods that require invasive procedures such as biopsy or surgery. Association studies of PARP-1 expression by IHC with prognosis and outcome have demonstrated mixed results, suggesting inconsistency of staining procedures and antibodies (25-28). Indeed, there lacks a validated clinical IHC staining protocol for PARP-1 that can be widely and robustly applied in clinical practice (28). Furthermore, approaches based on tissue sampling inadequately assess the potential heterogeneity of PARP-1 expression in disseminated EOC, a stage of disease highly relevant to PARPi therapy.

Radiotracer technology for the noninvasive imaging of PARP-1 could theoretically overcome the limitations of IHC by quantitatively assessing global PARP-1 expression in primary and disseminated disease $(31,32) .\left[{ }^{18} \mathrm{~F}\right]$ FluorThanatrace $\left(\left[{ }^{18} \mathrm{~F}\right] \mathrm{FTT}\right)$ is a radiolabeled small-molecule PARPi that is currently approved for clinical use under an investigational new drug application at the University of Pennsylvania (Philadelphia Pennsylvania, USA) and Washington University (St. Louis, Missouri, USA) $(33,34)$. $\left[{ }^{18} \mathrm{~F}\right] \mathrm{FTT}$ and its iodinated analogue $\left[{ }^{125} \mathrm{I}\right] \mathrm{KX} 1$ have been shown to correlate with PARP-1 expression through a receptor-ligand effect that stems from their primary pharmacological mechanism of action $(16,35)$. As such, $\left[{ }^{18} \mathrm{~F}\right] \mathrm{FTT}$ and $\left[{ }^{125} \mathrm{I}\right] \mathrm{KX} 1$ quantify PARP-1 expression and have the ability to measure drug-target engagement of clinical PARPis by competing with one another for the NAD ${ }^{+}$binding pocket on the catalytic subdomain of PARP-1. Contrary to current methodologies that measure the biochemical product of PARP-1, poly(ADP-ribose) (PAR), this is a direct measurement of drug-target engagement.

In this work, we validate the preclinical rationale for measuring PARP-1 expression as a predictive biomarker of response to PARPis and report the first clinical trial studying PARP-1 expression with $\left[{ }^{18} \mathrm{~F}\right]$ FTT PET in EOC.

\section{Results}

CRISPR/Cas9 deletion of PARP1 in ovarian cancer cells. Using CRISPR/Cas9 gene editing, we mediated the deletion of PARP1 in 2 ovarian cancer cell lines, one with a BRCA1 mutation and another with BRCA1 promoter methylation (UWB1.289 and OVCAR8). OVCAR8 cells have been previously shown to have reduced BRCA-1 expression attributed to promoter methylation and are sensitive to DNA-damaging agents (36-38). Strikingly, the genetic deletion of PARP1 in EOC cells with a BRCA1 mutation or BRCA1 promoter methylation did not result in synthetic lethality, in that the cells were viable and grew in culture $(21,39)$. Identifying the mechanism of viability was beyond the scope of this work, but is being pursued. Using this system for PARP1 deletion, we achieved more than $90 \%$ reduction of PARP-1 expression in polyclonal populations of BRCA1 mutant (UWB1.289) and BRCA1 methyl- 


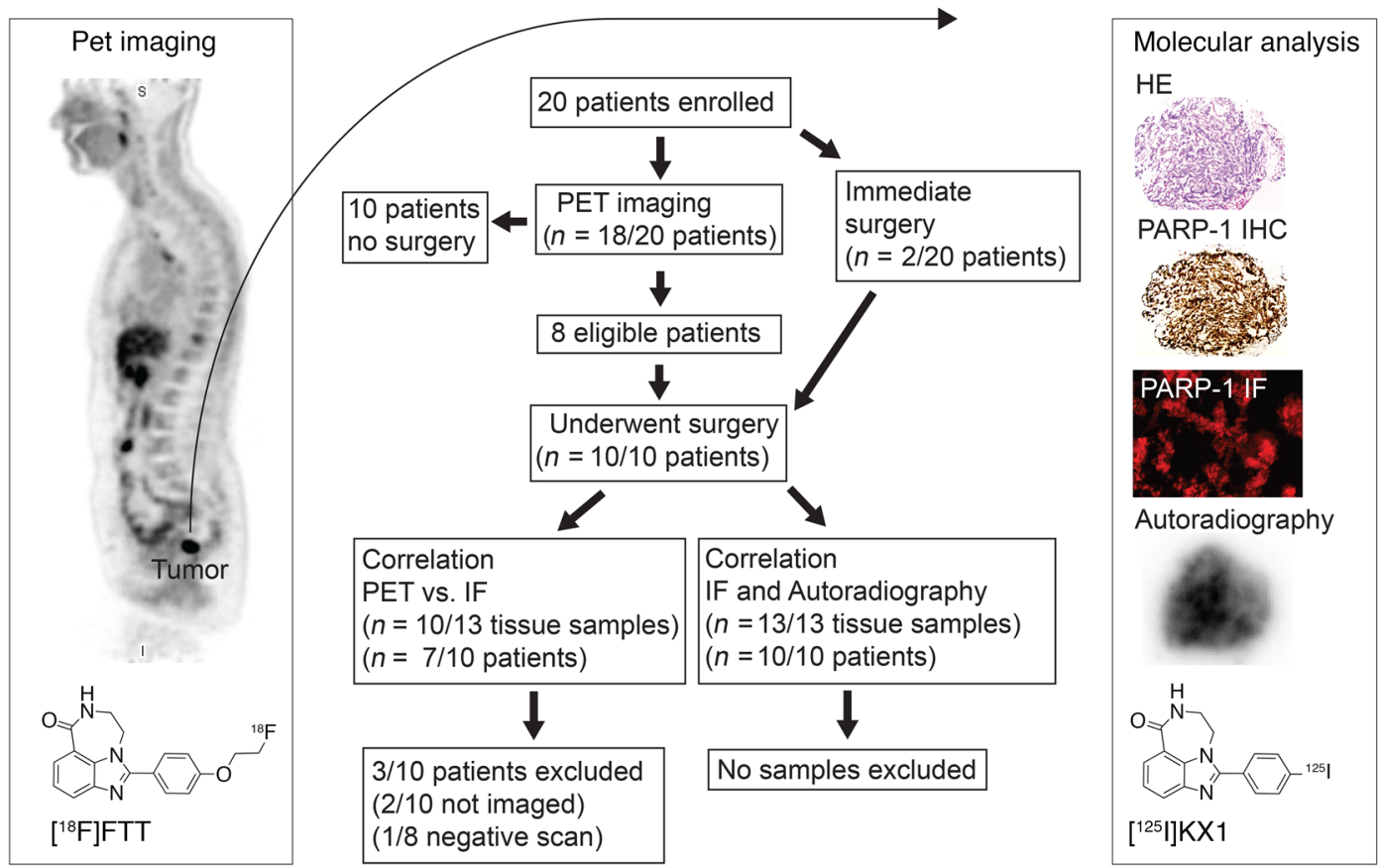

Figure 3. Diagram overview and flow chart of the pilot clinical trial of $\left[{ }^{18} \mathrm{~F}\right] \mathrm{FTT}$ PET/CT imaging in ovarian cancer. Ten patients who were enrolled and underwent PET imaging were excluded from this subanalysis due to lack of clinical tissue samples available for correlative studies.

ated (OVCAR8) ovarian cancer cells as measured by immunofluorescence (IF) and Western blot analysis (Figure 1, A and B, and Supplemental Figure 1, A and B; supplemental material available online with this article; https://doi.org/10.1172/JCI97992DS1). Cell microscopy studies showed that PARP-1 was indeed absent at the single-cell level in polyclonal populations (Figure $1 \mathrm{~A}$ and Supplemental Figure 1A). We also examined PARP-2 and PARP-3 expression by Western blot to investigate off-target effects of single-guide RNAs. We found no differences from control for PARP-2 or PARP-3 expression by Western blot analysis (Supplemental Figure 1B). Lastly, to determine whether PARP-1 expression varies among ovarian cell lines with and without BRCA dysfunction (Supplemental Table 1), we measured PARP-1 in multiple cell lines and demonstrated a dynamic range of expression (Supplemental Figure 2, A and B, and Supplemental Table 2).

$\left.{ }^{[25} I\right] K X 1$ radioligand binding measures differences in PARP-1 expression. Using the PARP1-KO cell lines along with several nonmodified EOC cell lines, we showed significant differences in the radioligand binding of $\left[{ }^{125} \mathrm{I}\right] \mathrm{KX} 1$ relative to PARP-1 expression (Figure 1C, Supplemental Figure 2C, and Supplemental Table 2). Most notably, the deletion of PARP1 reduced radioligand binding of $\left[{ }^{125} \mathrm{I}\right] \mathrm{KX} 1$ in both models. Furthermore, we observed that the radioligand binding of $\left[{ }^{125} \mathrm{I}\right] \mathrm{KX} 1$ was reduced in BRCA functional versus dysfunctional cells (Figure 1C, Supplemental Figure 2C, and Supplemental Table 2). The restoration of BRCA1 in UWB1.289 cells resulted in lower radioligand binding and corresponded to lower PARP-1 expression by IF and Western blot (Figure $1, \mathrm{~A}-\mathrm{C}$, and Supplemental Figure $2, \mathrm{~A}-\mathrm{C}$ ). These data support in vitro radioligand binding with $\left[{ }^{125} \mathrm{I}\right] \mathrm{KX} 1$ as a method to assay PARP-1 expression.

Loss of PARP1 blunts PARPi efficacy in ovarian cancer cell lines in vitro. The genetic deletion of PARP1 resulted in reduced DNA damage and sensitivity to PARPis in vitro. First, we examined isogenic UWB1.289 cells by IF after treatment with olaparib for 24 hours. We found no change in DNA damage measured by $\gamma \mathrm{H} 2 \mathrm{AX}$ foci formation following olaparib treatment in both BRCA1restored and PARP1-KO cells compared with DMSO controls. In contrast, $\gamma \mathrm{H} 2 \mathrm{AX}$ levels increased 2.6 times in olaparib-treated UWB1.289 cells compared with DMSO controls (Figure 1D). Similarly, the same effect was observed in OVCAR8 cells (Figure 1D). Next, the cytotoxicity evaluated for clinically used PARPis showed that the loss of PARP1 resulted in equal or greater resistance compared with restoring BRCA1 in UWB1.289 cells (Figure 1E, Supplemental Figure 2D, and Supplemental Table 3, A and B). Strikingly, a greater resistance in PARP1-KO cells compared with BRCA1-restored cells from parent controls was observed for the potent PARP trappers, niraparib and talazoparib. The loss of $P A R P 1$ resulted in greater than a 1,000-fold decrease in sensitivity to talazoparib, whereas restoring $B R C A 1$ had a lesser effect in UWB1.289 cells. OVCAR8 cells also became resistant to PARPis after loss of PARP1. No differences in sensitivity to cisplatin were observed in cell lines after loss of PARP1, indicating there was no change in overall sensitivity to DNA-damaging drugs. These data provide direct support for PARP-1 expression as a requirement for PARPi sensitivity in vitro.

Preclinical imaging of PARP-1 expression. The aforementioned findings demonstrate the need for dynamic, noninvasive, and quantitative monitoring of PARP-1 expression in patients selected for PARP inhibitor therapy. To this end, proof-of-concept studies were conducted in preclinical mouse patient-derived xenograft (PDX) models to confirm the in vitro radioligand binding studies and demonstrate the specificity of $\left[{ }^{18} \mathrm{~F}\right] \mathrm{FTT}$ for in vivo quantitative imaging of PARP-1. Significant differences were observed in radiotracer uptake in tumors before and after treatment with 
A

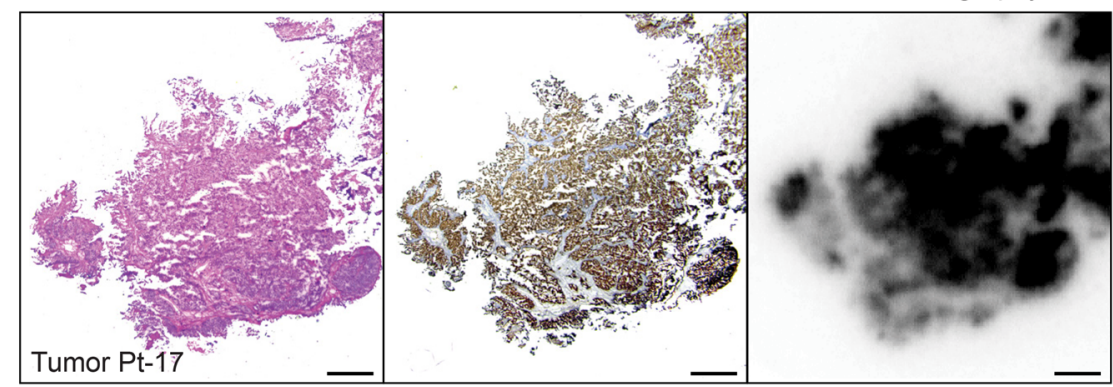

B

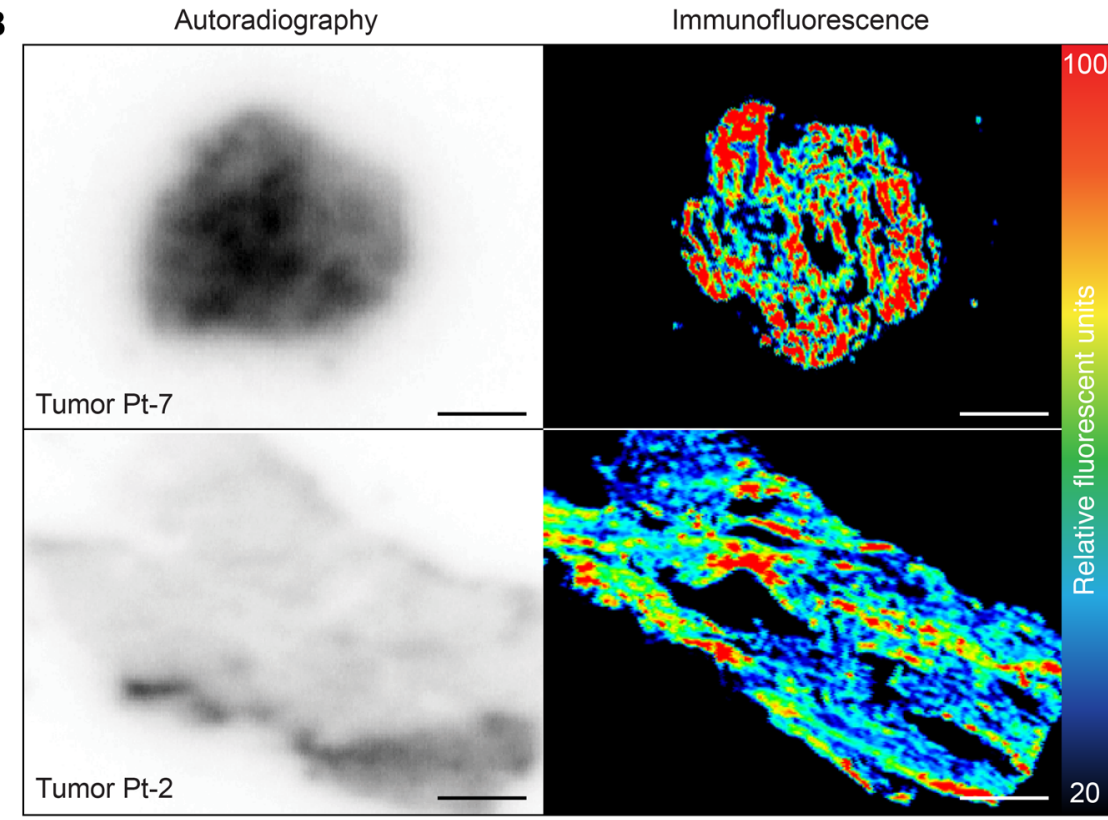

Figure 4. Immunohistochemistry and autoradiography analysis on clinical tissue. Distance bars represent $275 \mu \mathrm{M}$. (A) HE, PARP-1, and [125]]KX-1 autoradiograph on adjacent tissue sections showed colocalization between [ ${ }^{125}$ I] KX1 and PARP-1 C-IHC. (B) In vitro autoradiography showed a difference in PARP-1 expression that was also confirmed by PARP-1 f-IHC. Max intensity by autoradiograph was $0.28 \mathrm{vs}$. $0.20 \mu \mathrm{Ci} / \mathrm{mg}$ and fluorescent intensity of PARP-1 from whole-section f-IHC was 9.6 vs. 6.7 RFU.

olaparib. By administering olaparib $(50 \mathrm{mg} / \mathrm{kg}$ ) we observed a reduction in $\left.{ }^{18} \mathrm{~F}\right] \mathrm{FTT}$ tumor-to-muscle ratios, a semiquantitative measure of tracer binding in vivo (Figure 2A and Supplemental Figure $3 \mathrm{~A}$ ). In addition, tumor tissue analyzed from untreated and olaparib-treated mice by ex vivo autoradiography was used to confirm microPET findings (Figure 2B and Supplemental Figure 3B). Results between microPET imaging and ex vivo autoradiography were similar. Tumor-to-muscle ratios from microPET imaging before and after olaparib treatment were $4.2 \pm 0.32$ vs. $2.5 \pm 0.11$ (paired $t$ test, $P<0.0025, n=4$ ) (Figure 2C). In agreement with microPET results, ex vivo autoradiographs showed a significant difference between untreated and olaparib-treated groups (5.14 \pm 0.13 vs. $2.41 \pm 0.18$, unpaired $t$ test, $P<0.0001, n=2,10$ sections/ tumor) (Figure 2D). Autoradiographs also showed significant differences in tumor and muscle of untreated and olaparib-treated groups (unpaired $t$ test, $P<0.0001$ ) (Supplemental Figure 3C).

Clinical trial enrollment. In this trial, 20 patients were enrolled and 10 patients who underwent surgical debulking or biopsy were included in the study. From the 10 patients, 13 tis- sue specimens were collected for in vitro analysis. Of the 10 patients included in the study, 8 underwent PET/CT imaging (Figure 3). One of the 8 patients had a negative $\left[{ }^{18} \mathrm{~F}\right]$ FTT scan and was excluded from the $\left[{ }^{18} \mathrm{~F}\right]$ FTT PET versus PARP-1 IF correlation. PARP-1 immunohistochemistry and autoradiography on clinical specimens. Radioligand binding with $\left[{ }^{125} \mathrm{I}\right] \mathrm{KX} 1$ was found to colocalize with PARP-1 colormetric IHC (c-IHC) (Figure 4A) on adjacent tissue sections, supporting the specificity of the iodinated radiotracer for PARP-1. In addition, a spectrum of PARP-1 expression was observed by fluorescent IHC (f-IHC) and autoradiography (Figure 4B and Supplemental Figure 4), providing direct evidence for the wide range of PARP-1 expression in ovarian cancer. Interestingly, PARP-1 colocalized with both tumor cells and lymphocytes in the tumor microenvironment (Supplemental Table 4 and Supplemental Figure 5). PARP-1 expression was also positive in 2 lymph node samples, with residual disease on pathology, and costained with $\gamma \mathrm{H} 2 \mathrm{AX}$. Finally, positive staining for PARP-1 and p53 co-occurred in $77 \%(10 / 13)$ of tissue samples, whereas positive staining for PARP- 1 and $\gamma \mathrm{H} 2 \mathrm{AX}$ had 100\% (13/13) co-occurrence (Supplemental Table 4 and Supplemental Figure 5).

Clinical observations with $\left.{ }^{[18} \mathrm{F}\right] \mathrm{FTT}$ PET imaging. PET imaging demonstrated $\left[{ }^{18} \mathrm{~F}\right]$ FTT localized to areas of known EOC based on clinical $\left[{ }^{18} \mathrm{~F}\right]$ FDG PET/CT imaging with low bladder uptake, allowing for clear visualization of lesions within the pelvic region (Figure 5). Despite hepatobiliary excretion, $\left[{ }^{18} \mathrm{~F}\right]$ FTT uptake was discernible in omental disease in the abdomen (Figure 6). Differences in radiotracer uptake were seen between $\left[{ }^{18} \mathrm{~F}\right] \mathrm{FTT}$ and $\left[{ }^{18} \mathrm{~F}\right] \mathrm{FDG}$ PET, which is commonly used for evaluating tumor response and recurrence in EOC as well as identifying extrapelvic metastases and diagnosis. (40) Such differences suggest these radiotracers provide different and complementary information, concordant with their distinct molecular targets. For example, patient 2, who had completed 4 cycles of carboplatin and paclitaxel 2 weeks prior to imaging, showed multiple omental lesions by $\left[{ }^{18} \mathrm{~F}\right] \mathrm{FTT}$ PET imaging with maximum SUVs ranging from $5-8$ that were low on $\left[{ }^{18} \mathrm{~F}\right]$ FDG PET (Figure 6A, Figure 6B, and Supplemental Table 5). In follow-up, patient 2 showed disease progression within 4 months of therapy and was platinum resistant. In contrast, patient 11 had a BRCA1 mutation and showed low uptake on both $\left[{ }^{18} \mathrm{~F}\right] \mathrm{FTT}$ and $\left[{ }^{18} \mathrm{~F}\right] \mathrm{FDG}$ PET after 4 cycles of therapy (carboplatin and paclitaxel) and was platinum sensitive (Figure 6, C and D). These results suggest that $\left[{ }^{18} \mathrm{~F}\right]$ FTT PET provides unique molecular information as a biomarker of viable tumor tissue and could be used in the future to guide clinical management in addition to current biomarkers 
$\left[{ }^{18} \mathrm{~F}\right] \mathrm{FTT}$

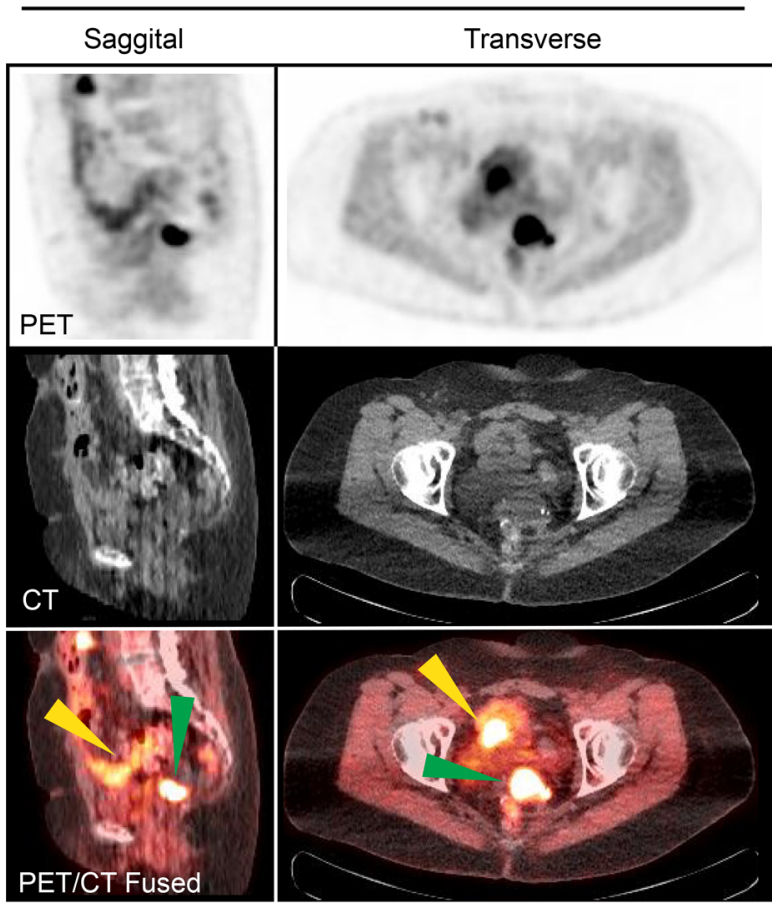

$\left[{ }^{18} \mathrm{~F}\right] \mathrm{FDG}$

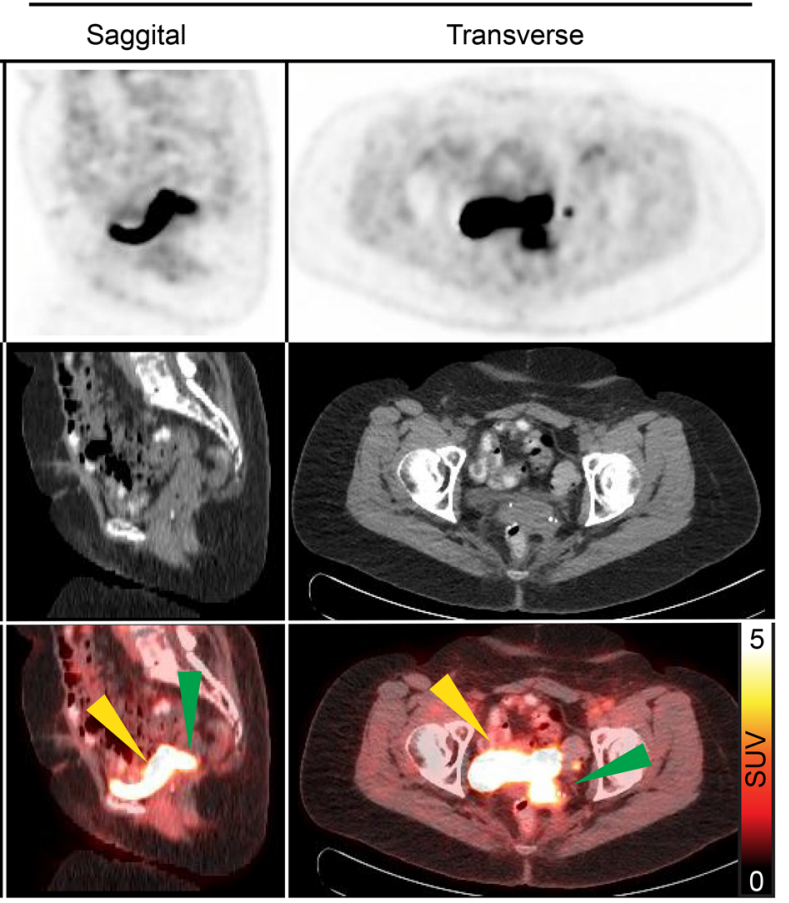

Figure 5. Clinical [ $\left.{ }^{18} \mathrm{~F}\right]$ FTT and [ $\left.{ }^{18} \mathrm{~F}\right]$ FDG PET/CT images of a patient with ovarian cancer with vaginal cuff lesion. Minimal radiotracer in the urinary bladder with $\left[{ }^{18} \mathrm{~F}\right] \mathrm{FTT}$ PET allowed for clear visualization of the lesion (green arrow) with no interference, despite some bowel uptake (yellow arrow on [ $\left.{ }^{18} \mathrm{~F}\right] \mathrm{FTT}$ image). Note excreted radiotracer in the bladder on $\left[{ }^{18} \mathrm{~F}\right] \mathrm{FDC}$ PET (yellow arrow on [18 $\left.\mathrm{F}\right] \mathrm{FDC}$ PET).

of metabolic activity. Intertumor heterogeneity of $\left[{ }^{18} \mathrm{~F}\right] \mathrm{FTT}$ was observed in patients (Supplemental Figure 6), further emphasizing a beneficial role of PET imaging compared with standard biopsy approaches for quantifying PARP-1 in local and disseminated disease.

Clinical $\left[{ }^{18}\right.$ F]FTT PET imaging positively correlates with PARP-1 expression in patient tumors. A spectrum of $\left[{ }^{18} \mathrm{~F}\right] \mathrm{FTT}$ uptake was seen in tumors, indicative of varying levels of PARP-1 expression (Supplemental Table 5). Maximum SUVs of tumors ranged from as low as 2 (background) to as high as 12 and a similar range of PARP-1 expression was also observed by immunofluorescence and autoradiography (Figure 7, A and B, and Supplemental Table 5). Since $\left[{ }^{18} \mathrm{~F}\right]$ FTT uptake is a marker of PARP-1 expression, this could translate to some tumors expressing up to 6 times as much PARP-1 as other tumors, with consequently higher drug-target densities for PARPis (Figure 7A). Further characterizing $\left[{ }^{18} \mathrm{~F}\right] \mathrm{FTT}$ as a measurement of PARP-1 expression, we found positive correlations between PARP-1 f-IHC and $\left[{ }^{18} \mathrm{~F}\right] \mathrm{FTT}$ PET imaging using linear regression analysis and calculating Pearson coefficients, in addition to PARP-1 f-IHC and $\left.{ }^{[25} \mathrm{I}\right] \mathrm{KX} 1$ autoradiography $\left(r^{2}=0.60,0.79, r=0.77,0.89\right)$ (Figure $7 \mathrm{~B}$ and Supplemental Table 5). As expected, there was no correlation observed among PARP-1 f-IHC, $\left[{ }^{18} \mathrm{~F}\right] \mathrm{FTT}$, or $\left[{ }^{125} \mathrm{I}\right] \mathrm{KX} 1$ autoradiography with $\left[{ }^{18} \mathrm{~F}\right] \mathrm{FDG}$, supporting distinct molecular imaging targets for $\left[{ }^{18} \mathrm{~F}\right] \mathrm{FTT}$ and $\left[{ }^{18} \mathrm{~F}\right] \mathrm{FDG}$.

\section{Discussion}

This translational work further supports PARP- 1 as the primary target for PARPis and describes the development of $\left[{ }^{18} \mathrm{~F}\right] \mathrm{FTT}$ as a noninvasive measure of regional tumor PARP-1 expression in patients with ovarian cancer. In vitro radioligand binding of $\left[{ }^{125} \mathrm{I}\right] \mathrm{KX} 1$, the radio-iodinated analog of $\left[{ }^{18} \mathrm{~F}\right] \mathrm{FTT}$, was previously reported (16) as a method to assay PARP-1 expression and was further confirmed in the present work. The genetic deletion of PARP1 was explored as a mechanism of PARPi resistance in a BRCA1 mutant and a BRCA1 methylated ovarian cancer cell line, and our findings support the notion that PARP-1 expression is necessary for PARPi sensitivity in vitro. Preclinical imaging of PARP-1 in mice with $\left[{ }^{18} \mathrm{~F}\right] \mathrm{FTT}$ demonstrated the specificity of the radiotracer for its molecular target. Lastly, $\left[{ }^{18} \mathrm{~F}\right] \mathrm{FTT}$ was studied in patients with EOC involved in a phase I trial. $\left[{ }^{18} \mathrm{~F}\right]$ FTT demonstrated a wide dynamic range of uptake correlating with in vitro measures of PARP-1 expression and the absence of correlation with $\left[{ }^{18} \mathrm{~F}\right] \mathrm{FDG}$. In addition, we confirmed the feasibility of $\left[{ }^{18} \mathrm{~F}\right] \mathrm{FTT}$ as a practical radiotracer to image lesions in disseminated omental disease despite bowel uptake. Given our small sample size, it is too early to remark on imaging nodal disease involvement with $\left[{ }^{18} \mathrm{~F}\right] \mathrm{FTT}$ although a recent preclinical study suggests uptake in malignant nodes is greater than normal lymph nodes in a diffuse large B cell lymphoma model (41). It is difficult to extrapolate between diseases, but we did observe a single case that showed nodal uptake on $\left[{ }^{18} \mathrm{~F}\right] \mathrm{FDG}$ but not $\left[{ }^{18} \mathrm{~F}\right] \mathrm{FTT}$ and was negative on pathology, which is consistent with inflammation. Together, these data are important first steps in establishing $\left[{ }^{18} \mathrm{~F}\right] \mathrm{FTT}$ as a novel noninvasive biomarker of PARP-1 expression and lay the foundation for future testing of $\left[{ }^{18} \mathrm{~F}\right] \mathrm{FTT}$ PET in parallel with PARPi clinical trials.

We explored the loss of drug-target expression as a mechanism of PARPi resistance by studying the effects of the genetic deletion of PARP1 in BRCA1 mutant and BRCA1 methylated cell 
A

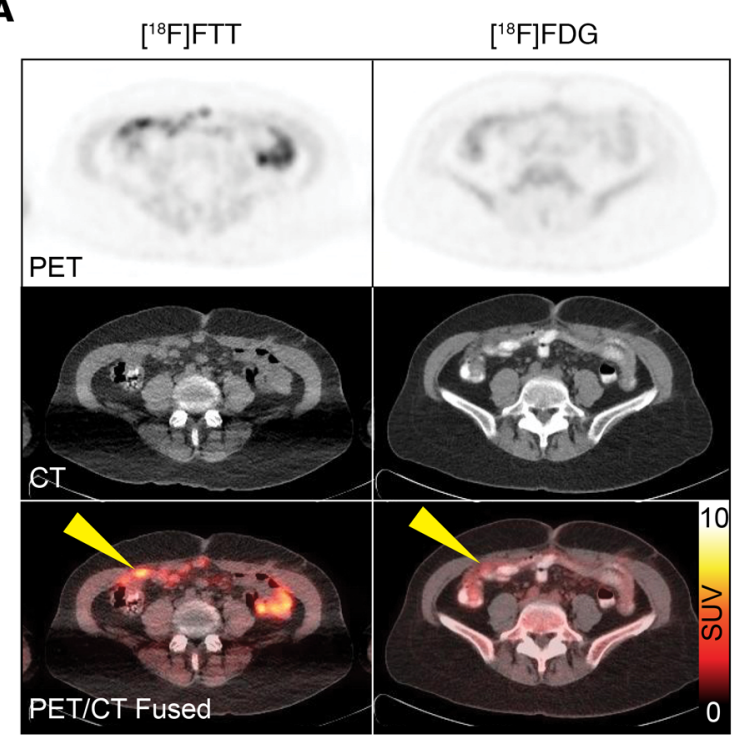

C

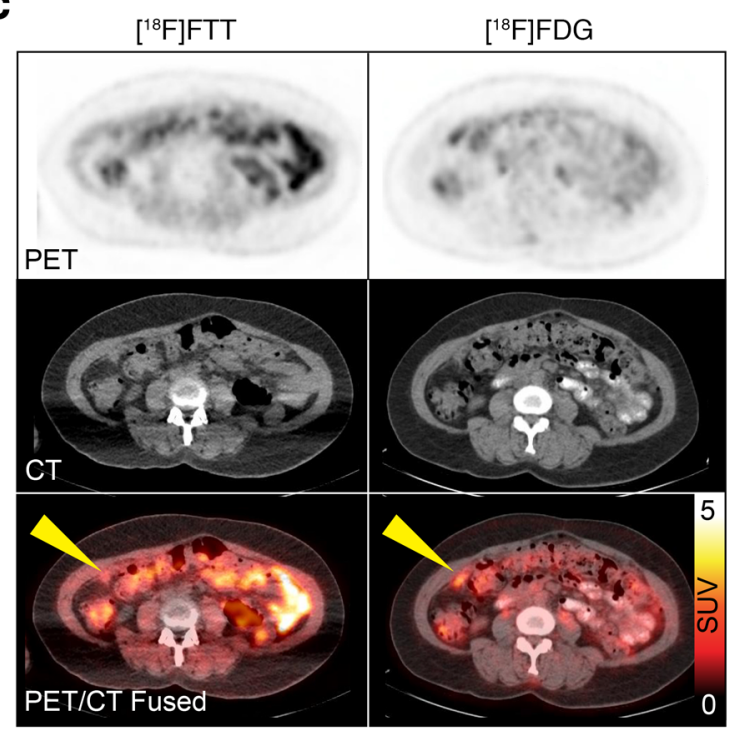

B

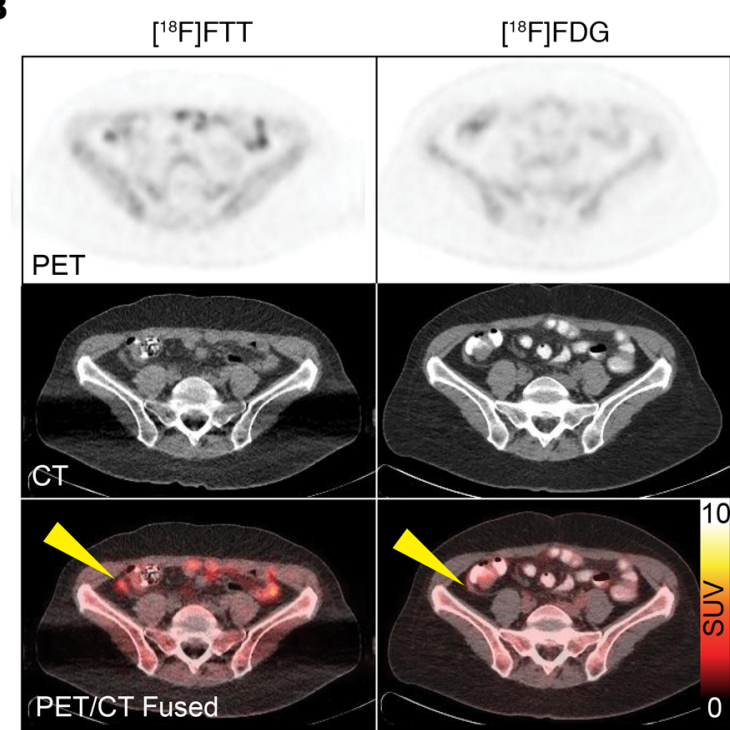

D

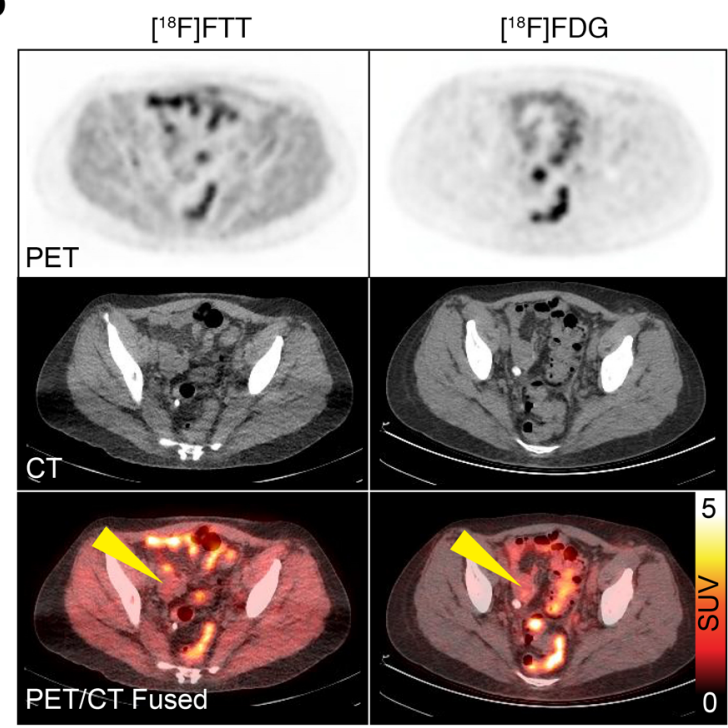

Figure 6. Patients 2 and 11 underwent $\left[{ }^{18} \mathrm{~F}\right] \mathrm{FTT}$ PET/CT imaging within 2 weeks of completing $\mathbf{4}$ cycles of carboplatin and paclitaxel. Omental metastases in patient 2 showed higher uptake of $\left[{ }^{18} \mathrm{~F}\right] \mathrm{FTT}$ than $\left[{ }^{18} \mathrm{~F}\right] \mathrm{FDG}$ with maximum SUVs of (A) 7.8 vs. 3.4 and (B) 5.1 vs. 2.0. Patient 2 was platinum resistant and relapsed within 4 months of therapy. Patient 11 showed low uptake on both [18 F]FTT and [ $\left.{ }^{18} \mathrm{~F}\right] \mathrm{FDC}$ with maximum SUVs of (C) 2.4 vs. 3.7 and (D) 2.3 vs. 2.9 . Patient 11 received 2 additional cycles of chemotherapy and was platinum sensitive. Yellow arrows indicate sites of disease.

lines. While loss of PARP1 in BRCA1 mutant or BRCA1 methylated cell lines has the potential to be synthetically lethal, we found that stable deletion of PARP1 by CRISPR/Cas9 resulted in polyclonal populations of cells in which more than $90 \%$ of clones lacked PARP-1 expression and grew in culture. It has been recently shown that OVCAR8 cells are capable of forming RAD51 foci, indicating functional homologous recombination, which could explain why loss of PARP1 was not synthetically lethal in this cell line (42). The observed viability with UWB1.289 cells is most likely due to the biological redundancy between PARP-1 and PARP-2 or PARP-3. Future studies are ongoing to assess PARP1/PARP2-KO and PARP1/PARP3-KO models to test this hypothesis. In addition, we intend to evaluate loss of PARP1 in isogenic BRCA2 mutants with and without reversion mutations, such as the PEO1/PEO4 cell lines, to test whether similar results are found. We also found that lack of PARP-1 expression prevented PARPis from inducing DNA damage, as measured by $\gamma \mathrm{H} 2 \mathrm{AX}$. These results are consistent with previous reports $(17-19,22,23)$. Loss of PARP1 most likely prevents the DNA damage caused by PARPi-induced PARP-1 trapping. This mechanistic understanding is highly relevant to PARPi therapy and our findings are consistent with what has been previously reported, showing PARP-1 as the primary target for PARPi-induced DNA damage $(3,14,15,17-19,23)$.

In further support, we also observed a greater reduction in PARPi sensitivity with loss of PARP-1 expression compared with BRCA1 restoration in the UWB1.289 isogenic cell lines when treated with the potent PARP trappers, niraparib and talazoparib. This observation may have translational implications, since olaparib, rucaparib, and niraparib were recently approved by the FDA as maintenance therapy in platinum-sensitive patients, regard- 
A
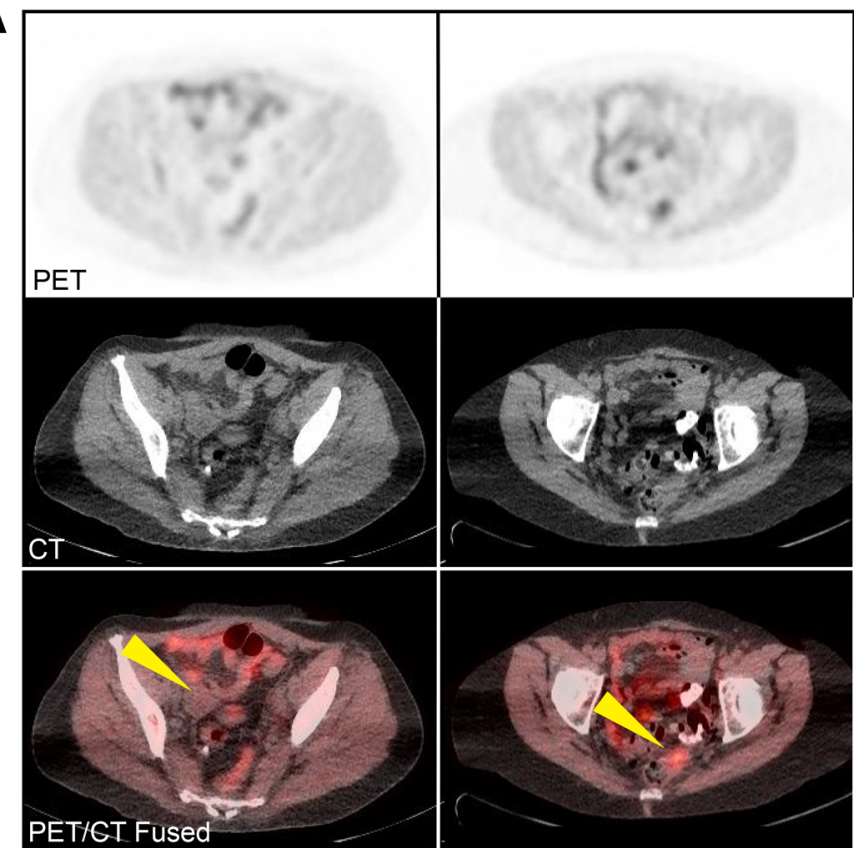

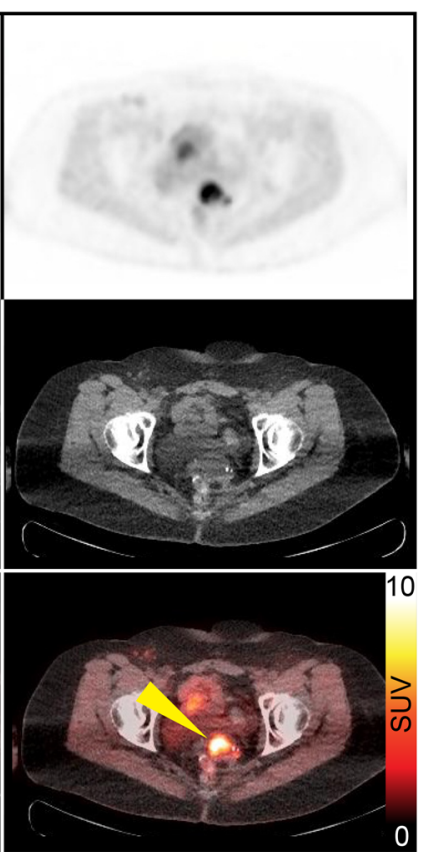

Figure 7. [ $\left.{ }^{18} \mathrm{~F}\right] \mathrm{FTT}$ PET imaging of PARP-1 in ovarian cancer patients and tissue correlates. (A) The spectrum of PARP-1 expression as determined by $\left[{ }^{18} \mathrm{~F}\right] \mathrm{FTT}$ PET/ CT imaging with maximum SUVs ranging from approximately 2 to 12. Yellow arrows indicate sites of disease. (B) We found a positive correlation between PARP-1 immunofluorescence versus [ $\left.{ }^{[8} \mathrm{F}\right] \mathrm{FTT}$ PET or $\left.\left[{ }^{125}\right]\right] \mathrm{KX} 1$ autoradiography (linear regression, $\left.r^{2}=0.60,0.79\right)$. No associations were observed between PARP-1 immunofluorescence, $\left[{ }^{18} \mathrm{~F}\right]$ FTT imaging, or [125]]KX1 autoradiography and $\left[{ }^{18} \mathrm{~F}\right] \mathrm{FDC}$.
B

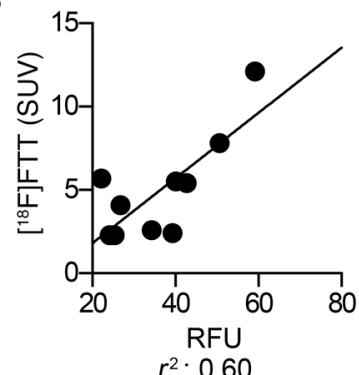

$r^{2}: 0.60$

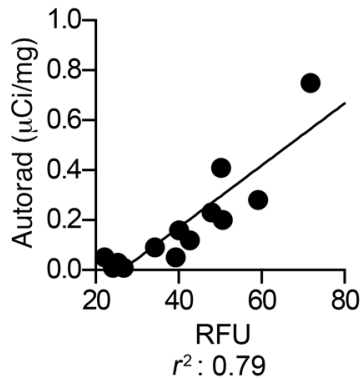

$r^{2}: 0.79$

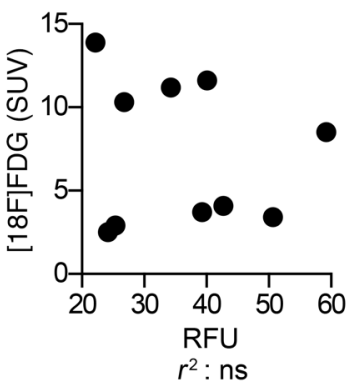

$r^{2}: \mathrm{ns}$ less of BRCA mutation status (6-8). These clinical reports suggest there is a need for predictive biomarkers for PARPi therapy that go beyond BRCA mutation and HRD testing. The direct measurement of the PARPi target, PARP-1 expression, described in this work offers a complementary approach that could strengthen genomic testing for predicting patient response to PARPi therapy.

We demonstrated that in addition to using radioligand binding as an in vitro assay of PARP-1 expression, the use of PET radiopharmaceuticals as an in vivo assay for PARP-1 is feasible in patients. This work reports the first clinical imaging trial in EOC using a PARP-1 radiotracer, $\left[{ }^{[8} \mathrm{F}\right] \mathrm{FTT}$, and preliminarily establishes $\left[{ }^{18} \mathrm{~F}\right]$ FTT as a noninvasive biomarker of PARP-1. We found a wide spectrum of PARP-1 expression in EOC measured by $\left[{ }^{[8} \mathrm{F}\right]$ FTT PET, with some tumors showing SUVs as high as 12 and as low as 2. Regardless of the exact mechanism in which PARPis induce anticancer effects, all current models require the drug target PARP-1 $(15,16$, $23,24)$. This signifies the importance of quantitatively assessing the target of PARPis directly at the site of action by measuring intratumoral PARP-1 in primary and metastatic disease. We have shown in preclinical imaging studies that $\left[{ }^{18} \mathrm{~F}\right] \mathrm{FTT}$ is capable of quantifying intratumoral PARPi-PARP-1 drug-target engagement. This is the only biomarker technology capable of noninvasively measuring PARP-1 and PARPi drug-target engagement directly in tumors, in contrast to surrogate assays such as peripheral blood test (43).
PARP-1 expression may also play a role in predicting inherent or incurred DNA damage burden to identify DNA repair deficiencies or even treatment response as a functional biomarker of $\operatorname{HRD}(44,45)$. We are currently entering several clinical trials in both breast and ovarian cancer at the University of Pennsylvania to directly evaluate $\left[{ }^{18} \mathrm{~F}\right] \mathrm{FTT}$ as a predictive and pharmacodynamic biomarker of PARPi therapy and other DNA-damaging chemotherapy. These studies should provide the impetus for multicenter clinical trials to fully evaluate the importance of PARP-1 expression as a biomarker for cancer therapy and the technological advantages of $\left[{ }^{18} \mathrm{~F}\right] \mathrm{FTT}$ for measuring intratumoral and intertumoral PARP-1 expression versus conventional IHC approaches.

\section{Methods}

Study design. The objective of this study was to highlight the significance of PARP-1 expression for PARP inhibitor therapy using preclinical models of ovarian cancer and perform the first PET imaging study of PARP-1 in ovarian cancer using $\left[{ }^{18} \mathrm{~F}\right] \mathrm{FTT}$ with histological correlates to confirm radiotracer uptake as a biomarker of PARP-1 expression. This goal was addressed by using CRISPR/Cas9 to delete PARP1 in models of ovarian cancer, which were then used to test PARPi efficacy in vitro. In addition, we used patient-derived orthotopic xenograft models of ovarian cancer to show proof of concept for $\left[{ }^{18} \mathrm{~F}\right] \mathrm{FTT}$ microPET imaging of PARP-1 and PARPi drug-target engagement in 
vivo. $\left[{ }^{18} \mathrm{~F}\right]$ FTT PET imaging of PARP-1 was performed in patients with ovarian cancer, and tumor specimens were retrieved at surgical debulking or biopsy for in vitro correlative analysis of PARP-1 using $\left.{ }^{[25} \mathrm{I}\right]$ KX1 autoradiography and PARP-1 f-IHC.

All in vitro experiments were carried out using a standard cell culture technique and repeated 3 independent times. The deletion of PARP1 with CRISPR/Cas9 was performed in 2 ovarian cancer cell lines. In addition, 3 unique guide RNAs were used to mediate the deletion of PARP1. Polyclonal populations of PARP1-KO cell lines were used to test PARPi efficacy in vitro. A sample size of 4 was selected for preclinical imaging studies to prevent bias greater than $10 \%$ for interpreting and analyzing microPET image results. No data from preclinical models was excluded from analyses.

For the clinical portion of this study, 20 patients with EOC were enrolled, 10 of whom had tissue available for in vitro analysis. Eight of those 10 underwent $\left[{ }^{18} \mathrm{~F}\right] \mathrm{FTT}$ imaging prior to subsequent standard clinical management (Figure 3). One of the 8 patients who underwent $\left[{ }^{18} \mathrm{~F}\right] \mathrm{FTT}$ had a negative PET image and was excluded from correlative analysis. A total of 13 tissue specimens was available from the 10 patients and these specimens were used for in vitro assays including PARP-1 f-IHC and $\left.{ }^{125} \mathrm{I}\right] \mathrm{KX} 1$ autoradiography (Supplemental Table 5). All tissue specimens from patients who underwent $\left[{ }^{18} \mathrm{~F}\right] \mathrm{FTT}$ PET imaging were included in correlations among $\left[{ }^{18} \mathrm{~F}\right] \mathrm{FTT}$ PET, $\left[{ }^{18} \mathrm{~F}\right]$ FDG, $\left.{ }^{125} \mathrm{I}\right] \mathrm{KX} 1$ autoradiography, and PARP-1 f-IHC. All tissue specimens collected from consenting patients were included in correlations between $\left[{ }^{125} \mathrm{I}\right] \mathrm{KX} 1$ and PARP-1 f-IHC. Patients with and without BRCA1 mutations were enrolled in this study (Supplemental Table 6). All experimental procedures for preclinical and clinical work are described in detail in the supplemental information.

CRISPR/Cas9 deletion of PARP1 in ovarian cancer cells. OVCAR8 cells were a gift from David M. Livingston (Dana Farber Cancer Institute, Boston, Massachusetts, USA). All other cell lines were available through ATCC or the Basser Center for BRCA (University of Pennsylvania, Philadelphia, Pennsylvania, USA) (Supplemental Table 1). PARP1 was deleted in UWB1.289 and OVCAR8 EOC cell lines that have reduced BRCA-1 expression through a deleterious mutation (UWB1.289) or promoter methylation (OVCAR8). These mutations are characterized elsewhere in the literature $(46,47)$. Using 3 unique guide RNAs (Supplemental Material), PARP1 deletion was mediated by Cas 9 in both cell lines, effectively producing 3 unique polyclonal PARP1-KO cell lines for each. Loss of PARP-1 expression was confirmed by IF cell microscopy and Western blot in each polyclonal population. Polyclonal populations were used in all experiments.

$\left.{ }^{125} I\right] K X 1$ radioligand binding measures differences in PARP-1 expression. Using multiple ovarian cancer cell lines, we performed in vitro pharmacological PARP-1 saturation radioligand binding studies to quantify PARP-1 expression. [ $\left.{ }^{125} \mathrm{I}\right] \mathrm{KX} 1$ was synthesized and in vitro radioligand binding studies were performed as previously described (16). Briefly, increasing concentrations of [ $\left.{ }^{[25} \mathrm{I}\right] \mathrm{KX} 1$ from $0.01-10 \mathrm{nM}$ were added to a 96-well plate in quadruplicate for each respective cell line and experiments were completed in triplicate.

Loss of PARP1 promotes PARPi resistance in ovarian cancer cell lines. Immunofluorescence was used to determine DNA damage induced by olaparib or DMSO control in ovarian cancer cell lines. Using 5 clinically used PARPis, we performed in vitro cell viability assays to determine changes in drug sensitivity based on loss of PARP-1 expression. The experimental procedures were adopted from previous work (16). Cispl- atin was also examined as a control to evaluate changes in sensitivity of DNA-damaging agents that occur after loss of PARP1. Cell viability was assessed using CellTiterGlo (Promega). Experiments were completed in duplicate 3 independent times.

Preclinical microPET imaging of PARP-1. Through serial $\left[{ }^{18} \mathrm{~F}\right] \mathrm{FTT}$ PET imaging studies, we evaluated PARP-1 drug-target engagement using the FDA-approved PARPi olaparib. PET imaging of PARP-1 drug-target engagement is a noninvasive methodology to directly assess PARP inhibition. Using 2 ovarian cancer patient-derived xenograft models in 15- to 18-week-old female NOD SCID mice provided from the Simpkins laboratory at the University of Pennsylvania (48), including a BRCA1 mutant and WT, we performed microPET imaging on a Phillips Medical Systems scanner $(n=4$ mice). Mice were imaged on day 1 and then olaparib was orally administered daily by gavage at a dose of $50 \mathrm{mg} / \mathrm{kg}$. Mice were then reimaged on day 4, 1 hour after the fourth dose of olaparib. A total of 4 doses of olaparib was administered between imaging studies to achieve steady state. Ex vivo autoradiography studies were performed on mice from PDX model WO-12-2 with and without olaparib treatment $(n=6)$. The differences in tumor-tomuscle ratios were measured and were representative of the percentage of PARPi drug-target engagement.

PARP-1 immunohistochemistry and autoradiography on clinical specimens. Colormetric immunohistochemistry was performed at the University of Pennsylvania Pathology Core for H\&E and biomarkers, including PARP-1, $\gamma \mathrm{H} 2 \mathrm{AX}$, p53, LCA, and AE1/3. These biomarkers were chosen due to their relevance to EOC. AE1/3 is a keratin marker and was used to identify tumor cells by c-IHC. In order to determine where in the tumor microenvironment PARP-1 was expressed, we costained with LCA, which is a pan lymphocyte marker. In addition, we analyzed common biomarkers present in high-grade serous EOC, including p53 and $\gamma \mathrm{H} 2 \mathrm{AX}$. Lastly, PARP- 1 was evaluated by f-IHC and $\left.{ }^{[25} \mathrm{I}\right] \mathrm{KX} 1$ autoradiography on adjacent sections to determine whether the 2 methods colocalize.

Clinical ${ }^{18}$ F]FTT PET imaging. The clinical trial included 2 imaging cohorts: a biodistribution cohort and a dynamic cohort. The patients included in this trial with pathologic correlates were all enrolled in the dynamic cohort. As such, only that protocol is reported in this section. Dynamic scanning was performed for approximately 60 minutes from the time of injection with a field-of-view over the abdomen/pelvis or to include a known site of tumor involvement. Static images from the skull base to mid-thigh were performed starting approximately 90 and 180 minutes after $\left[{ }^{18} \mathrm{~F}\right] \mathrm{FTT}$ injection; these static scans were optional at the discretion of the investigator or study physician. Static images obtained at approximately 90 minutes were used in the analysis. $\left[{ }^{18} \mathrm{~F}\right]$ FTT PET/CT imaging was performed on a Phillips Ingenuity PET/ CT scanner (Phillips Medical Systems). Images were reconstructed using standard reconstruction techniques and interpreted by trained radiology readers. Lesions were first located on a contemporaneous clinical $\left[{ }^{18} \mathrm{~F}\right]$ FDG PET/CT and then located on the $\left[{ }^{18} \mathrm{~F}\right] \mathrm{FTT}$ PET/CT. The maximum SUV was recorded for each scan.

Statistics. Data presented are mean \pm SEM unless otherwise noted. All graphs were produced and statistically analyzed using Prism version 6.0, GraphPad. The maximum number of PARP binding sites $\left(\mathrm{B}_{\max }\right)$ was calculated by an exponential nonlinear curve-fit 1-site binding hyperbola function. All statistical tests comparing the mean of 2 groups were 2 -sided. The comparisons of the means from more than 2 groups were performed using 1-way ordinary ANOVA. 
Dose-response curves were fitted using a nonlinear sigmoidal doseresponse curve, and effective calculations for $50 \%$ reduction in cell viability were calculated. Image analysis was performed using PMOD software, and tumor-to-muscle ratios were calculated. Preclinical microPET imaging studies comparing tumor uptake of radiotracer were analyzed using a paired, 2-tailed $t$ test. Autoradiography studies were analyzed using an unpaired 2-tailed $t$ test. We chose an $\alpha$ of 0.05 ( $95 \%$ confidence interval) and data were deemed significant if $P<0.05$. For correlations, the Pearson correlation coefficient was calculated and the data were considered statistically significant if $P<0.05$. To determine the ability of $\left[{ }^{18} \mathrm{~F}\right] \mathrm{FTT}$ PET maximum SUV to predict PARP-1 expression measured by f-IHC, we used a linear regression and results were considered statistically significant if different from zero.

Study approval. All animal studies were performed under protocols approved by the University of Pennsylvania Institutional Animal Care and Use Committee, Philadelphia, Pennsylvania, USA. Studies were conducted under guidelines for animal welfare provided by the NIH. This was an open-label, phase I trial of $\left[{ }^{18} \mathrm{~F}\right]$ FTT PET conducted under an approved IRB at the University of Pennsylvania, Philadelphia, PA, USA, and registered with the FDA as an expanded investigational new drug (clinical trial identifier: NCT02637934). All subjects consented to PET imaging and providing clinical tissue specimens for in vitro analysis.

\section{Author contributions}

MM designed experiments, performed experiments, collected data, analyzed data, and composed the manuscript. AP read PET scans, determined SUV values in lesions for PET tracers, analyzed data, and composed the manuscript. LS interpreted tissue histology and evaluated biomarker positivity. ES wrote the clinical protocol. KX synthesized precursors for radiotracers used. $\mathrm{CJH}$ analyzed preclinical PET imaging data. $\mathrm{CH}$ performed in vivo preclinical PET imaging studies. HK developed and produced patient-derived xenograft models. CCW performed ex vivo auto- radiography studies and analyzed data. HW designed CRISPR/ Cas9 guides for PARP-1 and performed in vitro PARP1-KO experiments. RD analyzed clinical PET images. MDF administered the radiotracer to patients and interpreted PET images. DAP administered the radiotracer to patients and interpreted PET images. RAG designed in vitro experiments and composed the manuscript. DAM administered the radiotracer to patients, designed experiments, interpreted the data, and composed the manuscript. FS designed patient-derived xenograft models, recruited patients, performed surgeries and biopsies, and composed the manuscript. RHM designed radiotracers and experiments and composed the manuscript. LLL designed experiments, analyzed data, recruited patients, lead the clinical trial, and composed the manuscript.

\section{Acknowledgments}

The authors would like to acknowledge the University of Pennsylvania PET center and cyclotron facility for clinical support and manufacturing of radiopharmaceuticals used in this study. The authors would like to specifically thank Mark A. Sellmyer and Elizabeth S. McDonald (Perelman School of Medicine, University of Pennsylvania) for their input regarding this work.

Address correspondence to: Mehran Makvandi, Division of Nuclear Medicine and Clinical Molecular Imaging, Department of Radiology, Perelman School of Medicine University of Pennsylvania, 231 South 34th Street, Philadelphia, Pennsylvania 19104, USA. Phone: 267.572.0621; Email: makvandi@pennmedicine. upenn.edu. Or to: Lilie L. Lin, Department of Radiation Oncology, University of Texas, MD Anderson Cancer Center, 1515 Holcombe Boulevard, Unit 1422, Houston, Texas 77030, USA. Phone: 713.563.2300; Email: 1llin@mdanderson.org.

LLL's present address is: Department of Radiation Oncology, University of Texas, MD Anderson Cancer Center, Houston, Texas, USA.
1. Siegel RL, Miller KD, Jemal A. Cancer statistics, 2016. CA Cancer J Clin. 2016;66(1):7-30.

2. Herzog TJ. The current treatment of recurrent ovarian cancer. Curr Oncol Rep. 2006;8(6):448-454.

3. Lord CJ, Ashworth A. PARP inhibitors: synthetic lethality in the clinic. Science. 2017;355(6330):1152-1158.

4. Konstantinopoulos PA, Ceccaldi R, Shapiro GI, D'Andrea AD. Homologous recombination deficiency: exploiting the fundamental vulnerability of ovarian cancer. Cancer Discov. 2015;5(11):1137-1154.

5. Mirza MR, Matulonis UA. Niraparib in recurrent ovarian cancer. $N$ Engl J Med. 2017;376(8):801-802.

6. Mirza MR, et al. Niraparib maintenance therapy in platinum-sensitive, recurrent ovarian cancer. N Engl J Med. 2016;375(22):2154-2164.

7. Coleman RL, et al. Rucaparib maintenance treatment for recurrent ovarian carcinoma after response to platinum therapy (ARIEL3): a randomised, double-blind, placebo-controlled, phase 3 trial. Lancet. 2017;390(10106):1949-1961.
8. Pujade-Lauraine E, et al. Olaparib tablets as maintenance therapy in patients with platinumsensitive, relapsed ovarian cancer and a BRCA1/2 mutation (SOLO2/ENGOT-Ov21): a double-blind, randomised, placebo-controlled, phase 3 trial. Lancet Oncol. 2017;18(9):1274-1284.

9. Tutt A, et al. Oral poly(ADP-ribose) polymerase inhibitor olaparib in patients with BRCA1 or BRCA2 mutations and advanced breast cancer: a proof-of-concept trial. Lancet. 2010;376(9737):235-244.

10. Tutt AN, et al. Exploiting the DNA repair defect in BRCA mutant cells in the design of new therapeutic strategies for cancer. Cold Spring Harb Symp Quant Biol. 2005;70:139-148.

11. Swisher EM, et al. Rucaparib in relapsed, platinum-sensitive high-grade ovarian carcinoma (ARIEL2 Part 1): an international, multicentre, open-label, phase 2 trial. Lancet Oncol. 2017;18(1):75-87.

12. Somlo G, et al. Efficacy of the PARP inhibitor veliparib with carboplatin or as a single agent in patients with germline BRCA1- or BRCA2-associated metastatic breast cancer: California Cancer
Consortium Trial NCT01149083. Clin Cancer Res. 2017;23(15):4066-4076.

13. de Bono J, et al. Phase I, dose-escalation, two-part trial of the PARP inhibitor talazoparib in patients with advanced germline BRCA1/2 mutations and selected sporadic cancers. Cancer Discov. 2017;7(6):620-629.

14. Pommier Y, O'Connor MJ, de Bono J. Laying a trap to kill cancer cells: PARP inhibitors and their mechanisms of action. Sci Transl Med. 2016;8(362):362ps17.

15. Pettitt SJ, et al. A genetic screen using the PiggyBac transposon in haploid cells identifies Parp1 as a mediator of olaparib toxicity. PLoS One. 2013;8(4):e61520.

16. Makvandi M, et al. A radiotracer strategy to quantify PARP-1 expression in vivo provides a biomarker that can enable patient selection for PARP inhibitor therapy. Cancer Res. 2016;76(15):4516-4524.

17. Ha HC, Snyder SH. Poly(ADP-ribose) polymerase is a mediator of necrotic cell death by ATP depletion. Proc Natl Acad Sci U S A. 1999;96(24):13978-13982. 
18. Heacock ML, Stefanick DF, Horton JK, Wilson SH. Alkylation DNA damage in combination with PARP inhibition results in formation of S-phasedependent double-strand breaks. DNA Repair (Amst). 2010;9(8):929-936.

19. Horton JK, Stefanick DF, Naron JM, Kedar PS, Wilson SH. Poly(ADP-ribose) polymerase activity prevents signaling pathways for cell cycle arrest after DNA methylating agent exposure. J Biol Chem. 2005;280(16):15773-15785.

20. Bryant HE, et al. Specific killing of BRCA2deficient tumours with inhibitors of poly(ADP-ribose) polymerase. Nature. 2005;434(7035):913-917.

21. Farmer $\mathrm{H}$, et al. Targeting the DNA repair defect in BRCA mutant cells as a therapeutic strategy. Nature. 2005;434(7035):917-921.

22. Murai J, et al. Trapping of PARP1 and PARP2 by Clinical PARP Inhibitors. Cancer Res. 2012;72(21):5588-5599.

23. Murai J, et al. Stereospecific PARP trapping by BMN 673 and comparison with olaparib and rucaparib. Mol Cancer Ther. 2014;13(2):433-443.

24. Hopkins TA, et al. Mechanistic dissection of PARP1 trapping and the impact on in vivo tolerability and efficacy of PARP inhibitors. Mol Cancer Res. 2015;13(11):1465-1477.

25. Godoy H, Mhawech-Fauceglia P, Beck A, Miller A, Lele S, Odunsi K. Expression of poly (adenosine diphosphate-ribose) polymerase and p53 in epithelial ovarian cancer and their role in prognosis and disease outcome. Int J Gynecol Pathol. 2011;30(2):139-144.

26. Brustmann H. Poly(adenosine diphosphateribose) polymerase expression in serous ovarian carcinoma: correlation with p53, MIB-1, and outcome. Int J Gynecol Pathol. 2007;26(2):147-153.

27. Gan A, Green AR, Nolan CC, Martin S, Deen S. Poly(adenosine diphosphate-ribose) polymerase expression in BRCA-proficient ovarian highgrade serous carcinoma; association with patient survival. Hum Pathol. 2013;44(8):1638-1647.

28. Hjortkjær M, Waldstrøm M, Jakobsen A, Kan- strup H, Søgaard-Andersen E, Dahl Steffensen $K$. The prognostic value of BRCA1 and PARP expression in epithelial ovarian carcinoma: immunohistochemical detection. Int J Gynecol Pathol. 2017;36(2):180-189.

29. Mazzotta A, Partipilo G, De Summa S, Giotta F, Simone G, Mangia A. Nuclear PARP1 expression and its prognostic significance in breast cancer patients. Tumour Biol. 2016;37(5):6143-6153.

30. Wu W, et al. Expression of PARP-1 and its active polymer PAR in prostate cancer and benign prostatic hyperplasia in Chinese patients. Int Urol Nephrol. 2014;46(7):1345-1349.

31. Knight JC, Koustoulidou S, Cornelissen B. Imaging the DNA damage response with PET and SPECT. Eur J Nucl Med Mol Imaging. 2017;44(6):1065-1078.

32. Carney B, Kossatz S, Reiner T. Molecular imaging of PARP. J Nucl Med. 2017;58(7):1025-1030.

33. Zhou D, et al. Synthesis, $\left[{ }^{18} \mathrm{~F}\right]$ radiolabeling, and evaluation of poly (ADP-ribose) polymerase-1 (PARP-1) inhibitors for in vivo imaging of PARP-1 using positron emission tomography. Bioorg Med Chem. 2014;22(5):1700-1707.

34. Michel LS, et al. PET of poly (ADP-Ribose) polymerase activity in cancer: preclinical assessment and first in-human studies. Radiology. 2017;282(2):453-463.

35. Edmonds CE, et al. [(18)F]FluorThanatrace uptake as a marker of PARP1 expression and activity in breast cancer. Am J Nucl Med Mol Imaging. 2016;6(1):94-101.

36. Busschots S, O'Toole S, O'Leary JJ, Stordal B. Carboplatin and taxol resistance develops more rapidly in functional BRCA1 compared to dysfunctional BRCA1 ovarian cancer cells. Exp Cell Res. 2015;336(1):1-14.

37. Stordal B, et al. BRCA1/2 mutation analysis in 41 ovarian cell lines reveals only one functionally deleterious BRCA1 mutation. Mol Oncol. 2013;7(3):567-579.

38. Choi YE, et al. Sublethal concentrations of 17-AAG suppress homologous recombination
DNA repair and enhance sensitivity to carboplatin and olaparib in HR proficient ovarian cancer cells. Oncotarget. 2014;5(9):2678-2687.

39. Ding $X$, et al. Synthetic viability by BRCA2 and PARP1/ARTD1 deficiencies. Nat Commun. 2016;7:12425.

40. Khiewvan B, et al. An update on the role of PET/ CT and PET/MRI in ovarian cancer. Eur J Nucl Med Mol Imaging. 2017;44(6):1079-1091.

41. Tang J, et al. Targeted PET imaging strategy to differentiate malignant from inflamed lymph nodes in diffuse large B-cell lymphoma. Proc Natl Acad Sci U S A. 2017;114(36):E7441-E7449.

42. Kondrashova O, et al. Secondary somatic mutations restoring RAD51C and RAD51D associated with acquired resistance to the PARP inhibitor rucaparib in high-grade ovarian carcinoma. Cancer Discov. 2017;7(9):984-998.

43. Rodler ET, et al. Phase I study of veliparib (ABT888) combined with Cisplatin and Vinorelbine in advanced triple-negative breast cancer and/ or BRCA mutation-associated breast cancer. Clin Cancer Res. 2016;22(12):2855-2864.

44. Li D, et al. A novel crosstalk between BRCA1 and poly (ADP-ribose) polymerase 1 in breast cancer. Cell Cycle. 2014;13(21):3442-3449.

45. Wu X, et al. FASN regulates cellular response to genotoxic treatments by increasing PARP-1 expression DNA repair activity via NF-кB SP1 [published online ahead of print October 24, 2016]. Proc Natl Acad Sci U S A. doi: 10.1073/ pnas.1609934113.

46. Mitra AK, et al. In vivo tumor growth of highgrade serous ovarian cancer cell lines. Gynecol Oncol. 2015;138(2):372-377.

47. DelloRusso C, Welcsh PL, Wang W, Garcia RL, King MC, Swisher EM. Functional characterization of a novel BRCA1-null ovarian cancer cell line in response to ionizing radiation. Mol Cancer Res. 2007;5(1):35-45.

48. George E, et al. A patient-derived-xenograft platform to study BRCA-deficient ovarian cancers. JCI Insight. 2017;2(1):e89760. 